\title{
Radiation Hardness Test of a Silicon Detector under Radiation Dose Rate of Nuclear Power Plant for In-Containment Coolant Leakage Detection System
}

\author{
Jongheon Kim ${ }^{1}$, Kihong Pak ${ }^{1}$, Junesic Park ${ }^{1}$ and Yong Kyun Kim ${ }^{1 *}$ \\ ${ }^{1}$ Department of Nuclear Engineering, Hanyang University, Seoul, 04763, and the Republic of Korea \\ "Corresponding author`s e-mail : ykkim4@ hanyang.ac.kr
}

\begin{abstract}
An influence evaluation by background radiation on a silicon detector, which will be used to detect a coolant leakage, and installed in a containment building of a nuclear power plant, was performed and the result was discussed. The detector that consists of a silicon sensor and preamplifier mounted in a shielding structure which composed of a $5 \mathrm{~cm}$ lead cylinder will be installed in an annulus zone that is influenced by background radiation (neutron and gamma ray) from an operation of a nuclear reactor. Absorbed dose rates on a silicon sensor and preamplifier were calculated as $2.15 \mathrm{mGy} / \mathrm{hr}$ and 1.05 mGy/hr, respectively, by Monte Carlo N-Particle (MCNP) simulation. Data of background radiation had referred to a Final Safety Analysis Report (FSAR) of a nuclear power plant in the Republic of Korea.

A silicon sensor and preamplifier were irradiated by a Co60 gamma radiation source equipped in a facility of Korea Atomic Energy Research Institute Advanced Radiation Technology Institute (KAERI ARTI) of the Republic of Korea. A Po-210 alpha source was used as a check source to evaluate a state of a function of the detector during gamma irradiation. Absorbed dose rates were about $22.92 \mathrm{mGy} / \mathrm{hr}$ and 6.6 $\mathrm{mGy} / \mathrm{hr}$ on silicon sensor and preamplifier, respectively. Before and during gamma irradiation, a count rates from the check source wasn't changed (from $18.4 \mathrm{cps}$ to $18.4 \pm 0.2 \mathrm{cps}$ after irradiation), and any degradations of function also weren`t observed. Even more harsh condition than calculated dose rates referred by the condition of background radiation of in-containment, the silicon detector maintained the ability of function of charged particles detection. Based on the result, it has been demonstrated that a silicon detector is a suitable detector for detecting charged particles from a leaked coolant even during interfered by the background radiation of a primary system of a nuclear power plant.
\end{abstract}

Keywords - Silicon detector, Irradiation test, Coolant leakage detection system

\section{INTRODUCTION}

A silicon detector, which has a thin depletion layer, has a characteristic that has small reactivity with gamma ray, and has high reactivity with charged particles. Therefore, the detector could be considered an option to use in a nuclear power plant that has a high dose of background gamma radiation during an operation. Therefore, we had suggested the silicon detector as a radiation detector of a project for developing a detection system of coolant leakage less than 0.5 gallons per minute in one hour $(0.5 \mathrm{gpm} / \mathrm{hr})$ [1]

The annulus zone of a nuclear power plant, in which the detector would be installed, has high dose background radiation during a plant`s operation. In the case of Advanced Power Reactor-1400 (APR-1400), the most advanced reactor model of the Republic of Korea, a design criterion of a dose rate at the location is $10 \mathrm{mSv} / \mathrm{hr}$. The detection system would be needed to have the ability to detect coolant leakage under high dose background radiation conditions. To protect the detector from background radiation, a $5 \mathrm{~cm}$ thickness lead cylinder structure was composed [1]. Even though the absorbed doses on the silicon sensor and preamplifier were dramatically reduced because of the structure, background radiation could impact the detector such as interfering signal, etc.

There are many studies to investigate a radiation hardness of a silicon detector especially specific conditions such as using as a detector for accelerators, or very high dose conditions to verifying damage of the detector $[2,3]$. However, there is no study to investigate the influence of a silicon detector under dose rate conditions of a nuclear power plant.

In this study, to investigate the effects of background radiation on the detector during an operation of the reactor, absorbed dose rates to the silicon sensor and preamplifier were calculated through MCNP simulation. Based on the results of the simulation, a gamma irradiation test was conducted at the KAERI ARTI facility using a Co-60 gamma source.

\section{MATERIAL AND METHOD}

\section{A. Simulation}

To determine the dose rate for exposure on a silicon sensor and preamplifier, MCNP simulation was used. The calculated absorbed doses in the annulus zone on the silicon sensor and preamplifier were about $2.15 \mathrm{mGy} / \mathrm{hr}$ and $1.05 \mathrm{mGy} / \mathrm{hr}$, respectively. Data of background radiation had referred Final Safety Analysis Report (FSAR) of a nuclear power plant in the Republic of Korea [4]. Using the data and MCNP simulation, fluxes of background radiation in an annulus zone were derived. Fig.1 shows the fluxes of gamma and neutron background radiation in the annulus zone of the nuclear power plant. Fig.2 shows the geometry of MCNP simulation to calculate absorbed doses. To calculate the intensities of background radiations, the source section was set up as F4 Tally, and outputs of F4 Tally was matched with the data of Fig.1. 

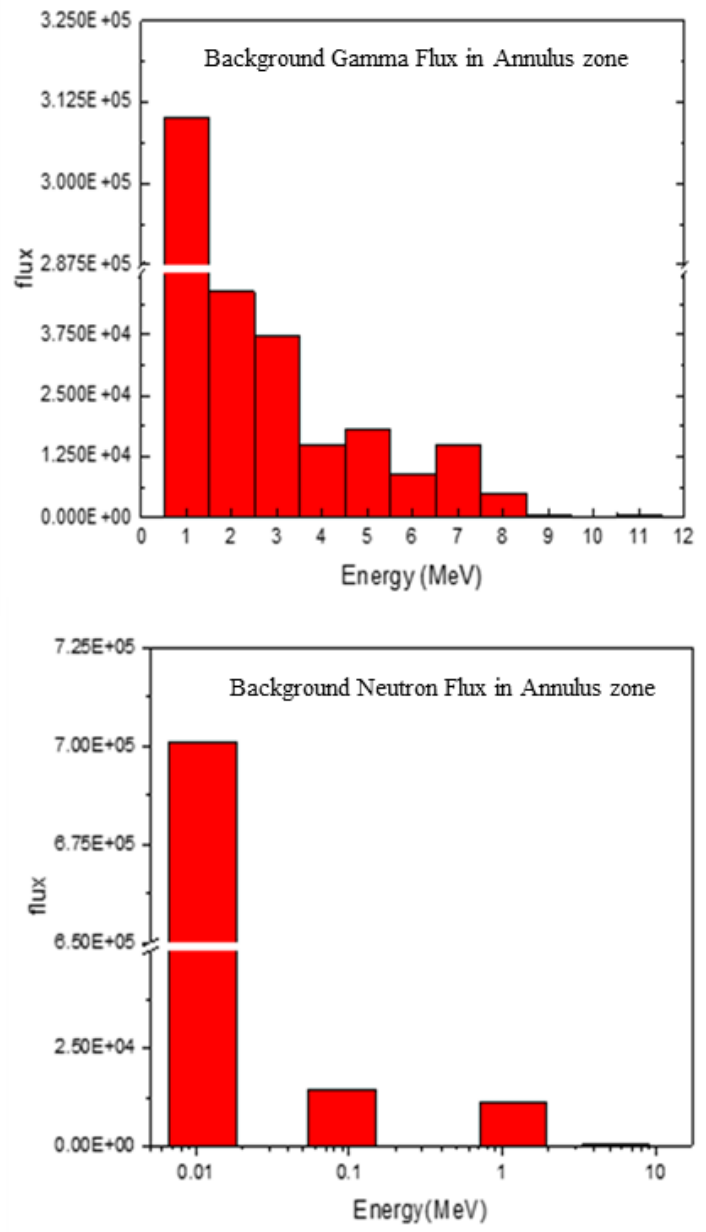

Fig. 1. The background radiation in an annulus zone of a nuclear power plant (top) gamma (bottom) neutron

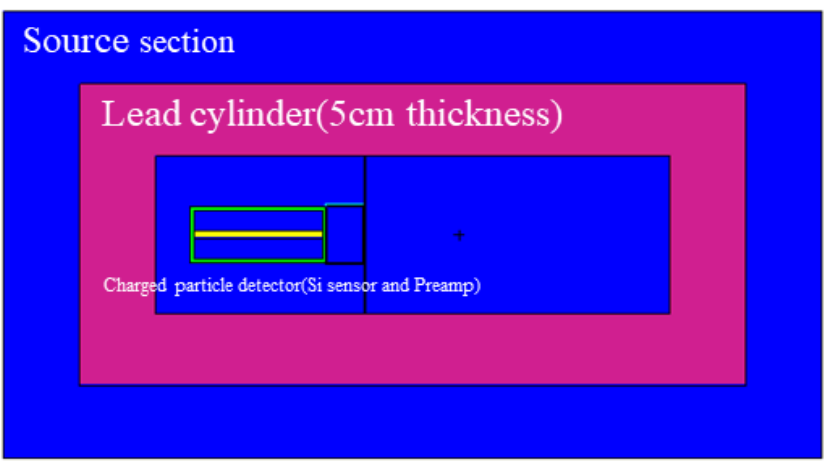

Fig. 2. MCNP geometry to calculate the absorbed dose rates on silicon sensor and preamplifier

\section{B. Experiment setting}

To investigate the influence of radiation exposure similar absorbed dose with an annulus zone of a nuclear power plant on a silicon sensor and preamplifier, an ULTRA ion implanted silicon sensor (ORTEC) was used. The silicon sensor had a 300 $\mu \mathrm{m}$ thickness of depletion depth and $1200 \mathrm{~mm}^{2}$ of an active area. As electronics devices, 142A preamplifier, 460 amplifier, 428

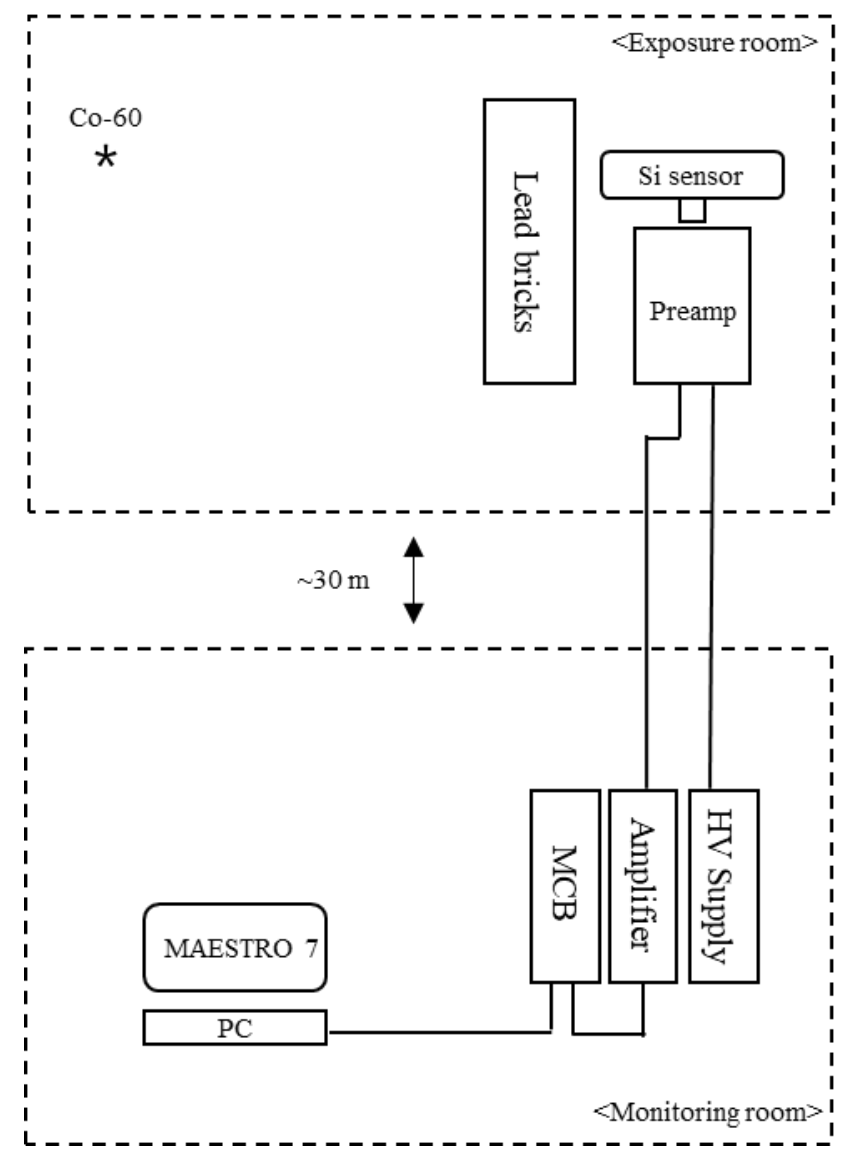

Fig. 3. Block diagram of the setting of the detector and electronics system

bias supply, $928 \mathrm{MCB}$, and MAESTRO ${ }^{\circledR} 7$ application software made by ORTEC were used for identifying spectra during radiation exposure.

The set value of electronics were $0.1 \mu \mathrm{sec}$ of shaping time, 50 of amplifier gain, $90 \mathrm{~V}$ of bias voltage, and 1024 channel of conversion gain.

The activity of the Co-60 gamma source for gamma irradiation was about $2.4 \mathrm{kCi}$. Therefore, to satisfy the situation of an absorbed dose rate of the detector in the annulus zone, the experiment was conducted using $10 \mathrm{~cm}$ lead bricks and sufficient distance from the source. Furthermore, to identify absorbed doses accumulated in the silicon sensor and preamplifier, alanine checkers were used. In addition, a Po-210 alpha source was used as a check source for identifying functional states of the silicon detector during gamma irradiation. Fig. 3 and Fig. 4 present a block diagram and the setting of the experiment.

Because the reliability of absorbed dose rate data by alanine checker could be reduced under several Gy/hr, an absorbed dose at the point before shielding by lead bricks was measured. After that, using MCNP simulation, absorbed dose rates were calculated. Fig. 5 shows the geometry to calculate the experimental absorbed dose rates for each component. 


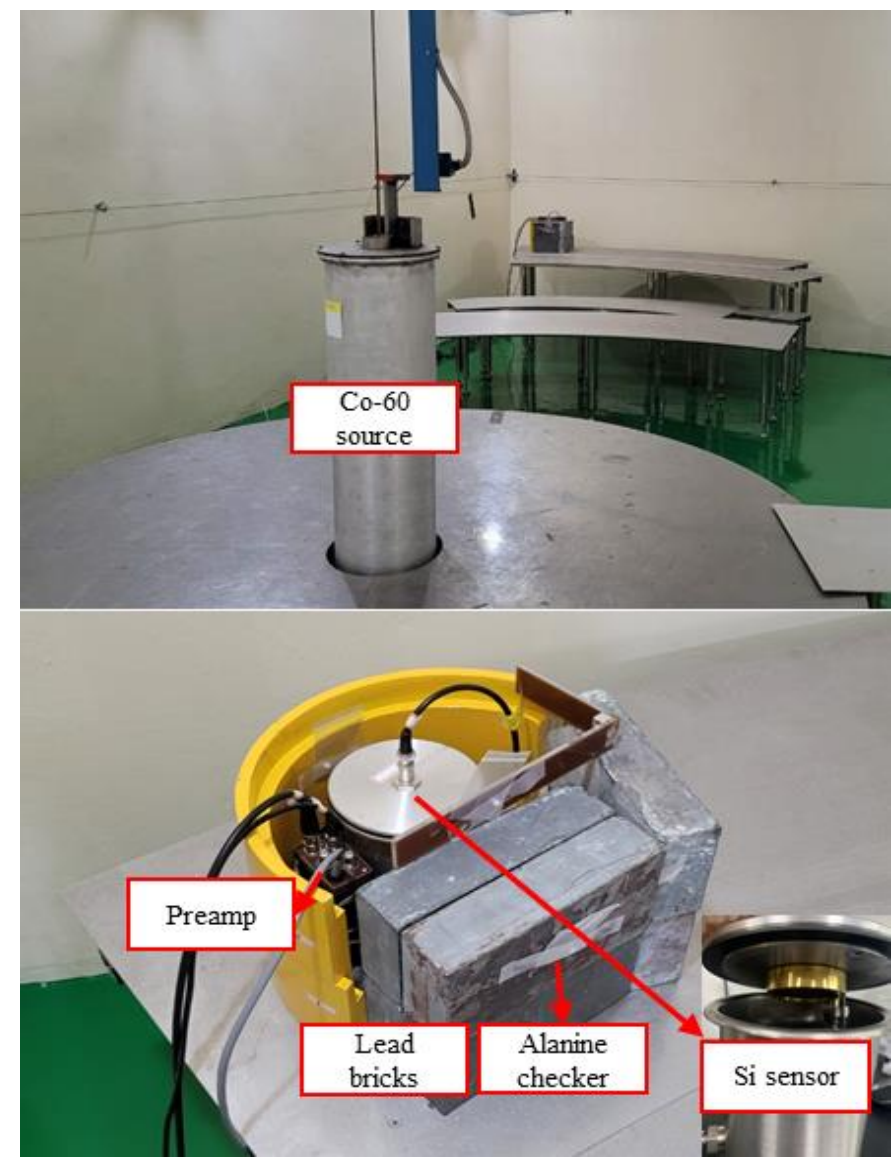

Fig. 4. Radiation hardness experiment on the silicon sensor and preamplifier at KAERI ARIT facility

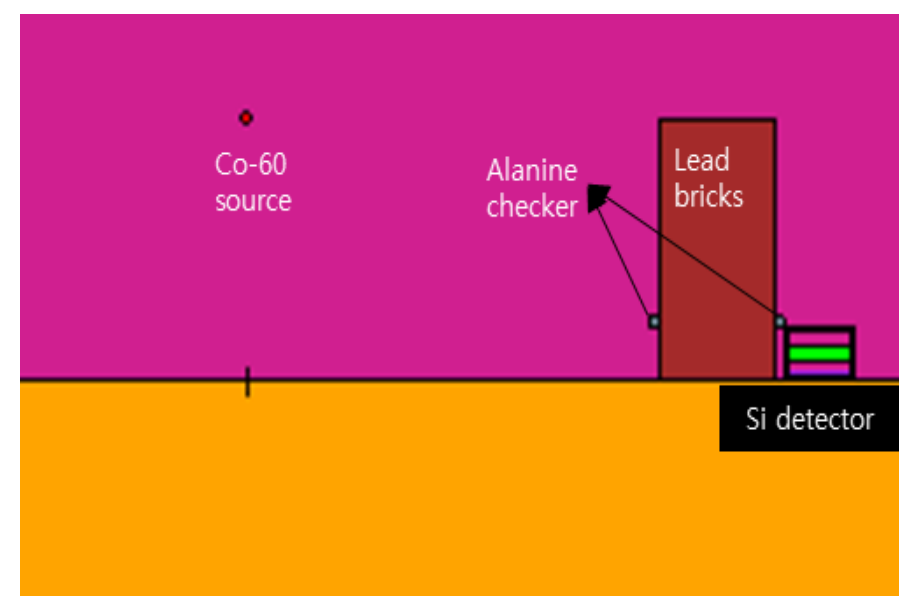

Fig. 5. MCNP geometry to calculate the experimental absorbed dose on the silicon sensor and premplifier

TABLE I

THE EXPERIMENTAL ABSORBED DOSE RATES ON EACH COMPONENT

\begin{tabular}{ccc}
\hline \hline Components & F6 Tally & Absorbed dose rate \\
\hline Silicon & $3.5207 \mathrm{E}-08$ & $22.92 \mathrm{mGy} / \mathrm{hr}$ \\
Preamp & $1.0107 \mathrm{E}-08$ & $6.6 \mathrm{mGy} / \mathrm{hr}$ \\
Alanine & $4.1311 \mathrm{E}-06$ & $2,690 \mathrm{mGy} / \mathrm{hr}$ \\
\hline \hline
\end{tabular}

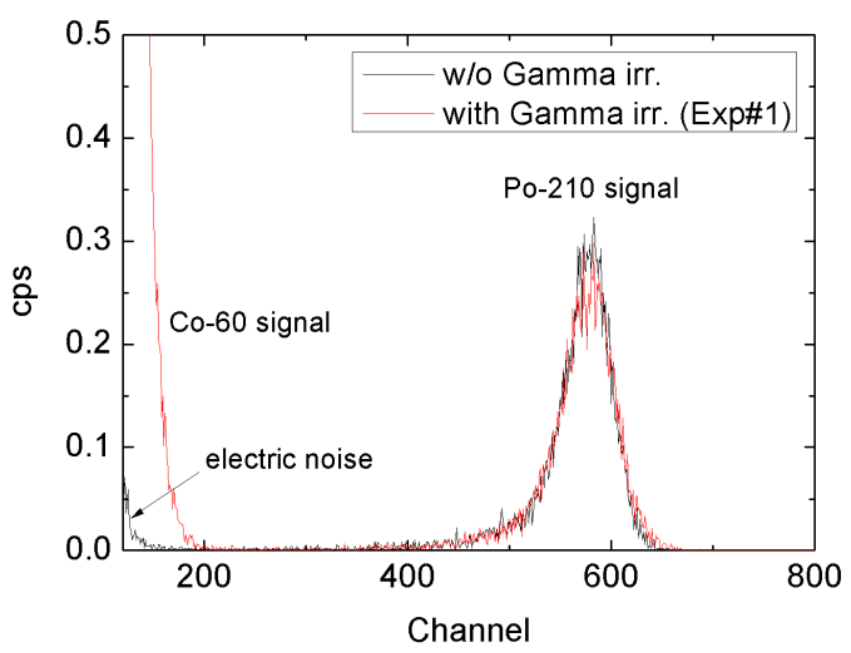

Fig. 6. The alpha and gamma signals before and during gamma irradiation

\section{RESULT AND DISCUSSION}

The experimental absorbed dose rates on the silicon sensor and preamplifier were $22.92 \mathrm{mGy} / \mathrm{hr}$, and $6.6 \mathrm{mGy} / \mathrm{hr}$, respectively. Table I shows the results of F6 Tally and absorbed dose rates at the silicon sensor, preamplifier, and alanine checker. The absorbed dose rate of the alanine checker is experimental data, and the absorbed dose rates of the silicon sensor and preamplifier are calculated value using the experimental data.

During gamma irradiation on the silicon sensor and preamplifier, a total of 12 repeated measurements were performed at 10-minute intervals to obtain spectra and to investigate the influences of gamma irradiation on charged particle detection. Fig. 6 shows the spectra before and during gamma irradiation. The first experiment shows that the counts of alpha peak slightly decrease compared with before gamma irradiation. In case of high dose background radiation conditions, the gross counts could be increased because of signals by the background radiation, and it also could make increase dead time. Therefore, the peak signal of alpha could be decreased by the increased the dead time. In the following experiments, however, there was no degradation by the dead time. In other words, the situation of the experiment in which gamma absorbing about 6 to $23 \mathrm{mGy} / \mathrm{hr}$ on the silicon detector, is not a condition to decrease the functional states of the detector.

Fig. 7 and Fig. 8 show the alpha and gamma spectra during the 12 times experiments, and the alpha counts fluctuation during gamma irradiation. There are no remarkable fluctuations and degradation of alpha counts in the spectra, such as shifting of alpha peaks, and a remarkable decrease of alpha counts, during gamma irradiation. The average count rates of alpha before and during irradiation are 18.4, and 18.4 \pm 0.2 , respectively.

Even though the experiment's conditions were harsher than the conditions of the annulus zone of a nuclear power plant, the 


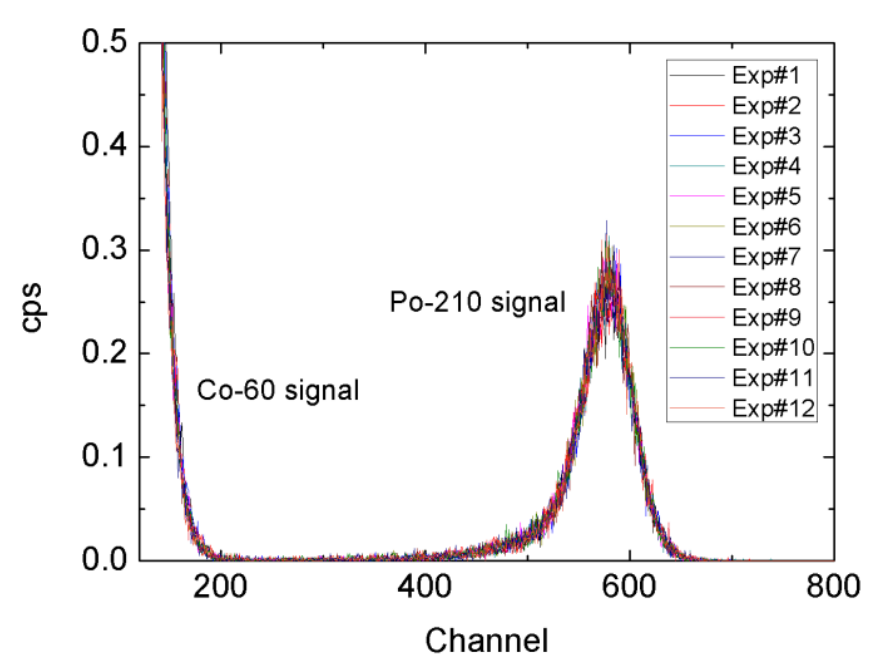

Fig. 7. The alpha spectra of the total of 12 repeated experiment that performed at 10-minute intervals

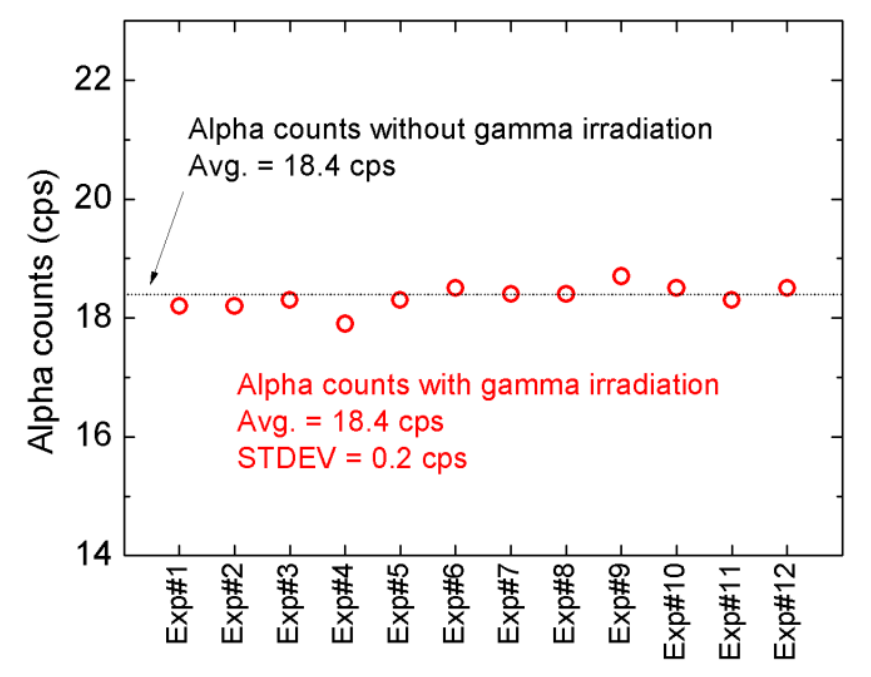

Fig. 8. Alpha counts before and during gamma irradiation

functional states of the silicon detector for detecting the ability of charged particles were maintained. Therefore, it is verified that the silicon detector could be used as a detector at the annulus zone for detecting charged particles from leaked coolant.

\section{CONCLUSION}

To develop a coolant leakage radiation detection system, the radiation hardness test under the condition of dose rates of the annulus zone of a nuclear power plant was conducted and the result was discussed. The absorbed dose rates on the silicon sensor and preamplifier were $22.92 \mathrm{mGy} / \mathrm{hr}$, and $6.6 \mathrm{mGy} / \mathrm{hr}$, respectively, which are higher compared with under the simulated condition of the annulus zone of the nuclear power plant. In other words, in more harsh conditions than the annulus zone, the detector remains functional integrity. Especially, it is verified that the ability to detect charged particles, such as alpha, could be maintained even during gamma irradiation. Based on the result, it has been demonstrated that a silicon detector is a suitable detector for detecting charged particles from a leaked coolant even during interfered by the background radiation in the annulus zone of the nuclear power plant.

\section{ACKNOWLEDGMENT}

This work was supported by Korea Institute of Energy Technology Evaluation and Planning (KETEP) grant funded by the Korea government (MOTIE) (Development of a real time detection system for unidentified RCS leakage less than 0.5 gpm)

\section{REFERENCES}

[1] J. Y. Jang, T. schaarschmidt, Y. K. Kim, "Feasibility Study of Beta Detector for Small Leak Detection inside the Reactor Containment", Journal of Radiation Protection and Research, Vol. 43, No. 4, pp.154159(2018)

[2] P. J. Sellin, J. Vaitkus, "New materials for radiation hard semiconductor detectors", Nuclear Instruments \& Methods in Physics Research Section A, Vol.557, Issue 2, pp. 479-489(2006)

[3] S. Metzger, H. Henschel, O. Köhn, and W. Lennartz, "Silicon Carbide Radiation Detector for Harsh Environment", IEEE Transactions on Nuclear Science, Vol. 49, Issue 3, pp.1351-1355(2002)

[4] Korea Hydro \& Nuclear Power Co., Ltd, Final Safety Analysis Report of Shin-Kori Nuclear Unit 3,4, Republic of Korea(2015) 